\title{
The comparative analysis of the three dilatation techniques in percutaneous nephrolithotomy: Which one is safer?
}

\author{
Aytac Sahin, Fatih Uruc \\ Department of Urology, Fatih Sultan Mehmet Research and Training Hospital, Istanbul, Turkey.
}

\begin{abstract}
Summary Objectives: Introduction of the "access sheath" is one of the most important steps of the percutaneous nephrolithotomy (PNL) intervention. In creating the access tract, various dilatators (balloon, metal) are used and different need-based dilatation tools were developed. In this study, we aimed to compare the mechanical Amplatz dilatation $(A D)$, balloon dilatation (BD) and one-shot dilatation (OSD) methods in a retrospective manner.

Methods: A total of 182 patients (127 males and 55 females), who underwent PNL surgery in Urology Department of Fatih Sultan Mehmet Research and Training Hospital between January 2016 and September 2018, were included in this study. Results: The average age was $47.34 \pm 12.68$ years (age range 15-80) and average BMI was $27.15 \pm 5.01 \mathrm{~kg} / \mathrm{m}^{2}$ (range between 17.12 and $40.75 \mathrm{~kg} / \mathrm{m}^{2}$ ). There was a prominent difference in terms of operation duration $(p=0.032)$. Meaningful difference was found among the groups in terms of dilation fluoroscopy time $(p=0.001)$, with a notable shorter time in OSD group than the others $(p<0.05)$. Beside this, there was no difference between the $A D$ and $B D$ groups in terms of fluoroscopy times ( $p>0.05)$. Also, there was no difference among the groups by Clavien complication rate $(p>0.05)$. There was a prominent difference among the groups in terms of hemoglobin decrement $(p=0.012 ; p<0.05)$. The hemoglobin decrease in $O S D$ group was significantly lower than in $A D$ and $B D$ groups $(p<0.05 ; p<0.01)$. On the contrary, there was no meaningful difference between $A D$ and $B D$ groups with this regard ( $p>$ 0.05).
\end{abstract}

Conclusions: As a result, we have concluded that the use of OSD modality in PNL interventions could be superior to other methods with respect to its feasibility, cost-effectiveness, shorter radiation exposure / fluoroscopy time and it could be a preferable way of treatment especially in developing countries.

KEY WORDS: Percutaneous nephrolithotomy; Dilatation techniques; One shot dilatation.

Submitted 30 May 2019; Accepted 26 July 2019

\section{INTRODUCTION}

Urolithiasis is a frequently encountered illness with prevalence rates highly variable according to region and population worldwide (1). Urolithiasis treatment can be applied by using conservative, medical or surgical methods according to the patient's status and characteristics of the stone (2).

Percutaneous nephrolithotomy (PNL) can be defined as kidney excision and removal of the kidney stones by inserting an access sheath between the kidney and subcutaneous region. PNL has become an important alternative to open surgery approach in urolithiasis treatment, with respect to its minimal invasive feature (3).

In current clinical practice, extracorporeal shock wave lithotripsy (ESWL), PNL, retrograde intrarenal surgery (RIRS), combined approach of these methods and other laparoscopic methods are used. Due to minimal complication rates, fast elimination of big stones, quicker recovery, short hospitalization periods, increased quality of life and minimal loss of working capacity, the European Association of Urology (EAU) has recommended the PNL method as primary option for treatment of kidney stones larger than two $\mathrm{cm}$ in diameter (2).

Introduction of the "access sheath" is one of the most important steps of the PNL intervention. In creating the access tract, various dilatators (balloon, metal) are used and different need-based dilatation tools were developed. In this study, we aimed to compare the mechanical Amplatz dilatation (AD), balloon dilatation (BD) and one-shot dilatation (OSD) methods in retrospective manner.

\section{MATERIALS AND NeTHOdS}

A total of 182 patients (127 males and 55 females), who underwent PNL surgery at Urology Department of Fatih Sultan Mehmet Research and Training Hospital between January 2016 and September 2018, were included in this study. All participants were thoroughly examined and evaluated prior to surgical intervention. Patients who have positive bacterial contamination in urinary culture, hemorrhagic diathesis condition or any systemic comorbidity were previously treated according to the diagnosed disorder and subsequently included to the study. Only individuals with serious coagulopathy were excluded. Retrograde pyelography imaging was taken from all the patients. PNL surgery was applied to all patients in prone position with assisted retrograde pyelography imaging. After calyceal access to kidney, AD method was applied to 66 patients, BD method in 55 patients and OSD in 61 patients. Average age, calculi diameter, body mass index, operation duration, radiation 
exposure during the surgical intervention, hemoglobin decrement rate, Clavien complication score and treatment costs were compared in the three groups, Radiation exposure time elapsed during dilatation was defined as the time period between the insertion of the 18 Gauge needle and the placement of Ampltaz sheath. Surgical operation was carried out by two urology specialists.

In OSD method, renal cavities were punctured by using 18 Gauge needle and guide-wire was inserted into collecting system after urinary flow was observed. Following this initial step, co-axial dilator of $10 \mathrm{~F}$ of diameter was pushed forward through the guide-wire. Amplatz dilators up to $30 \mathrm{~F}$ were further pushed into the kidney with rotation movement and access sheath was placed over Amplatz dilator, which enables a wide entry tract for nephroscopic examination.

\section{Statistical analysis}

SPSS Statistics 22 (IBM SPSS, Turkey) program was used for statistical analysis of the data obtained. Descriptive statistical data were obtained (average, standard deviation, frequency), Kruskall Wallis test was used to compare non-normal parameters for more than 2 groups and Mann Whitney U testwas used to compare differences between two independent groups.

\section{RESULTS}

The mean age was $47.34 \pm 12.68$ years (range 15-80) and average BMI was $27.15 \pm 5.01 \mathrm{~kg} / \mathrm{m}^{2}$ (range between 17.12 and $40.75 \mathrm{~kg} / \mathrm{m}^{2}$ ) (Table 1 ). There was a prominent difference in terms of operation time $(p=0.032)$. Significant difference was found among the groups in terms of dilation fluoroscopy time $(\mathrm{p}=0.001)$, with a notable shortening in OSD group than the others ( $\mathrm{p}<$ $0.05)$. Beside this, there was no difference between the $\mathrm{AD}$ and $\mathrm{BD}$ groups in terms of fluoroscopy times ( $\mathrm{p}>$ 0.05). Also, there was no difference among the groups

Table 1.

Demographic data.

\begin{tabular}{|lccc|}
\hline & $\begin{array}{c}\text { Mechanical } \\
\text { dilatation }\end{array}$ & $\begin{array}{c}\text { Balloon } \\
\text { dilatation }\end{array}$ & $\begin{array}{c}\text { One-shot } \\
\text { dilatation }\end{array}$ \\
\hline Average age (years) & 48.86 & 45.94 & 46.5 \\
\hline Male/female $(\mathrm{n})$ & $48 / 18$ & $39 / 16$ & $40 / 21$ \\
\hline Body mass index $\left(\mathrm{kg} / \mathrm{m}^{2}\right)$ & 27.06 & 27.28 & 27.13 \\
\hline Stone surface area $\left(\mathrm{cm}^{2}\right)$ & 5.4 & 4.8 & 4.28 \\
\hline
\end{tabular}

Table 2.

Evaluation of the parameters according to the operations carried out.

\begin{tabular}{|lcccc|}
\hline & $\begin{array}{c}\text { Mechanical } \\
\text { dilatation } \\
\text { Mean } \pm \text { SD (median) }\end{array}$ & $\begin{array}{c}\text { Balloon } \\
\text { dilatation } \\
\text { Mean } \pm \text { SD (median) }\end{array}$ & $\begin{array}{c}\text { One-shot } \\
\text { dilatation } \\
\text { Mean } \pm \text { SD (median) }\end{array}$ & P \\
\hline Surgery time (mins) & $117.73 \pm 49.53(120)$ & $104.92 \pm 35.86(120)$ & $99.1 \pm 39.34(90)$ & $0.032^{*}$ \\
\hline Scopy time in dilatation $(\mathrm{sec})$ & $30.05 \pm 6.87(28)$ & $27.02 \pm 4.06(27)$ & $25.39 \pm 3.23(25)$ & $0.001^{* *}$ \\
\hline Clavien score & $1.35 \pm 0.62(1)$ & $1.29 \pm 0.58(1)$ & $1.15 \pm 0.36(1)$ & 0.197 \\
\hline Hemoglobin level $(\mathrm{mg} / \mathrm{dL})$ & $1.44 \pm 1.3(1)$ & $1.39 \pm 1.24(1)$ & $0.92 \pm 0.44(0,8)$ & $0.012^{*}$ \\
\hline Kruskall- Wallis Test & $* p<0.05$ & ${ }^{* *}<0.01$ & & \\
\hline
\end{tabular}

for Clavien complication rates $(p>0.05)$. There was a prominent difference among the groups in terms of hemoglobin decrement ( $\mathrm{p}=0.012 ; \mathrm{p}<0.05)$. The hemoglobin decrease in OSD group was significantly lower than in $\mathrm{AD}$ and $\mathrm{BD}$ groups $(\mathrm{p}<0.05 ; \mathrm{p}<0.01)$. On the contrary, there was no statistically significant difference between the $\mathrm{AD}$ and $\mathrm{BD}$ groups with this regard ( $\mathrm{p}>$ 0.05) (Table 2).

\section{Discussion}

With the development of minimally invasive treatment of urinary calculi, PNL has become one of the main treatments for large kidney and upper ureteral stones (4). One of the most fundamental steps of PNL surgery is to establish safe and effective access. However, complications in this process such as tract dilation failure, hemorrhage and perforation of the renal parenchyma or collecting system are not uncommon (5).

The dilation of the nephrostomy tract is a central step of PNL intervention, and is usually performed by three dilation methods: Ssemi rigid fascial dilators (Amplatz) over an $8 \mathrm{~F}$ guide catheter, metal telescopic dilatators (MTD) and nephrostomy balloon dilatators (BD). Each dilation method has advantages and disadvantages. In the past, there have been many attempts and modifications to obtain the best results with minimal kidney damage (68 ). When it comes to the radiation exposure issue, $\mathrm{BD}$ and OSD methods have an obvious superiority to MD and $\mathrm{AD}$ methods, attributed to their easy-to-use aspect with one-pass dilation technique and shorter intervention time. In addition to this, there are also some cons of the latter mechanical methods including the displacement of guide-wire and access sheath during the multipass dilation entries, perforation in collecting tubule system and hemorrhage (9).

Although there is a general consensus that BD is a more reliable and efficient method than the metallic and plastic dilator use in the literature, it has been reported in a multi-centric study that there was longer mean operation time, more bleeding amount and higher transfusion rates with BD (10).

In a meta-analysis study in which four different dilation methods have been evaluated in 6820 patients submitted to PNL, it has been reported that OSD method requested shorter fluoroscopy time and less hemoglobin loss, when compared with the MTD method. In the same study, a statistically remarkable difference has been reported between BD and MTD in terms of blood transfusion rates and it has also been mentioned that BD had an advantage over MTD approach in terms of shorter intervention times and lower transfusion rate in patients who have not undergone open kidney surgery operation before. This meta-analysis research has also pointed out that OSD could be preferred in most patients that have to be operated by PNL (11). Likewise, we have observed less fluo- 
roscopy use and hemoglobin decrement in OSD method in the current study.

In a meta-analysis study including large series of participants, it has been reported that bleeding was significantly higher with balloon dilation method (9.4\%), when compared with telescopic/serial dilation (6.7\%). It has also been mentioned in the same study that there was a need for more transfusions for balloon dilation interventions (7.0\%) than telescopic/serial dilations (4.9\%) (12). Moreover, hemorrhage risk gets higher when a consecutive dilator is displaced to insert a larger one, because of the space remained after the removal. In some papers, single-increment dilation has been suggested as an easyto-apply and safe method to achieve better outcomes (13).

In a study by Frattini et al., it has been reported that there was a significant decrement in fluoroscopy time in comparison of the MTD, BD and OSD groups (14). Li et al. found that OSD was a more reliable method, because of shorter insertion and fluoroscopy times (15).

In another prospective series with 320 participants, Harrech et al. reported that OSD was also a reliable and feasible method, which could also be used in PNL procedures performed in supine position (16). It has been emphasized in the literature that tract dilation failure was a crucial issue, which could be encountered especially in cases with renal hypermobility condition. Harrech et al. reported the dilation tract failure rate as three percent (16). In our study, we did not had failures of access tract dilation with OSD orocedure.

Consequently, in our retrospective study, average fluoroscopy time and decrease in hemoglobin were found better by using OSD technique than $\mathrm{AD}$ and $\mathrm{BD}$.

Consequently our opinion is that OSD method is the safer and simpler technique.

\section{Conclusions}

As a result, we conclude that the use of OSD modality in PNL interventions could be superior to other methods with respect to its feasibility, cost-effectiveness, shorter radiation exposure/fluoroscopy times and it could be a preferable way of treatment especially in developing countries.

\section{REFERENCES}

1. Stoller ML. Urinary Stone disease; in Tanagho EA Mc Aninch JW 17th edition Smith's general urology, McGraw Hill Medical 2008.

2. Türk C, Skolarikos A, Neisius A, et al. Guidelines on Urolithiasis: European Association of Urology, 2019.

3. Peng PX, Lai SC, Ding ZS, et al. One-shot dilation versus serial dilation technique for access in percutaneous nephrolithotomy: a systematic review and meta-analysis. BMJ Open. 2019; 9:e025871.

4. Tomaszewski JJ, Smaldone MC, Schuster T, et al. Factors affecting blood loss during percutaneous nephrolithotomy using balloon dilation in a large contemporary series. J Endourol. 2010; 24:20711 .

5. Ozok HU, Sagnak L, Senturk AB, et al. A comparison of metal telescopic dilators and Amplatz dilators for nephrostomy tract dilation in percutaneous nephrolithotomy. J Endourol. 2012; 26:630-4.
6. Patil AV. A novel 5-part percutaneous access needle with guidewire technique (5-PANG) for percutaneous nephrolithotomy: our initial experience. Urology. 2010; 75:1206-8.

7. Baldwin DD, Maynes LJ, Desai PJ, et al. A novel single step percutaneous access sheath: the initial human experience. J Urol. 2006; 175:156-61.

8. Maynes LJ, Desai PJ, Zuppan CW, et al. Comparison of a novel one-step percutaneous nephrolithotomy sheath with a standard twostep device. Urology. 2008; 71:223- 7.

9. Hajiha M, Baldwin DD. New technologies to aid in percutaneous access. Urol Clin North Am. 2019; 46:225-243.

10. Yamaguchi A, Skolarikos A, Buchholz NP, et al. Operating times and bleeding complications in percutaneous nephrolithotomy: a comparison of tract dilation methods in 5,537 patients in the Clinical Research Office of the Endourological Society Percutaneous Nephrolithotomy Global Study. J Endourol. 2011; 25:933-9.

11. Dehong C, Liangren L, Huawei L, Qiang W. A comparison among four tract dilation methods of percutaneous nephrolithotomy: a systematic review and meta-analysis. Urolithiasis. 2013; 41:523-30.

12. Lopes T, Sangam K, Alken P, et al. Clinical Research Office of The Endourological Society Percutaneous Nephrolithotomy Study Group. The Clinical Research Office of the Endourological Society Percutaneous Nephrolithotomy Global Study: tract dilation comparisons in 5537 patients. J Endourol. 2011; 25:755-62.

13. Xiong J, Shi Y, Zhang X, et al. Chinese one shot dilation versus sequential fascial dilation for percutaneous nephrolithotomy: a feasibility study and comparison.Urol J. 2019; 16:21-26.

14. Frattini A, Barbieri A, Salsi P, et al. One shot: A novel method to dilate the nephrostomy access for percutaneous lithotripsy. J Endourol. 2001; 15:919-23.

15. Li Y, Yang L, Xu P, et al. One-shot versus gradual dilation technique for tract creation in percutaneous nephrolithotomy: a systematic review and meta-analysis. Urolithiasis 2013; 41:443-8.

16. El Harrech Y, Abakka N, El Anzaoui J, et al. One-shot dilation in modified supine position for percutaneous nephrolithotomy: experience from over 300 cases. Urol J. 2014; 11:1575-82.

\section{Correspondence}

Aytac Sahin, MD (Corresponding Author)

draytacsahin@gmail.com

Fatih Uruc, MD

drfatihguruc@gmail.com

Department of Urology, Fatih Sultan Mehmet Research and Training

Hospital, Istanbul, Turkey 